\title{
Vanishing theorems for the $\bmod p$ cohomology of some simple Shimura varieties
}

\author{
Teruhisa Koshikawa
}

Research Institute for Mathematical Sciences, Kyoto University, Kitashirakawa Oiwakecho, Sakyo-ku, Kyoto 606-8502, Japan; E-mail: teruhisa@kurims.kyoto-u.ac.jp.

Received: 24 December 2019; Revised: 18 May 2020; Accepted: 22 June 2020

2020 Mathematics Subject Classification: Primary-11G18; Secondary-11F33, 11F80

Keywords and phrases: Hecke algebras; Galois representations; Shimura varieties

\begin{abstract}
We show that the mod $p$ cohomology of a simple Shimura variety treated in Harris-Taylor's book vanishes outside a certain nontrivial range after localizing at any non-Eisenstein ideal of the Hecke algebra. In cases of low dimensions, we show the vanishing outside the middle degree under a mild additional assumption.
\end{abstract}

\section{Introduction}

Let $F$ be a $\mathrm{CM}$ field that contains an imaginary quadratic field $\mathcal{K}$. Let $G$ be a unitary similitude group that is associated with a division algebra $B$ with center $F$ of dimension $n^{2}$ and an involution of the second kind, so that it gives rise to Kottwitz's simple Shimura variety $X_{K}$ for a fixed sufficiently small level $K$ defined over the reflex field $E$.

Fix a prime number $p$. Let $m$ be a system of Hecke eigenvalues appearing in $H_{\mathrm{ett}}^{i}\left(X_{K, \bar{E}}, \overline{\mathbf{F}}_{p}\right)$ for some $i$. Caraiani and Scholze [2] constructed a semisimple Galois representation

$$
\rho_{\mathfrak{m}}: \Gamma_{F}:=\operatorname{Gal}(\bar{F} / F) \rightarrow \mathrm{GL}_{n}\left(\overline{\mathbf{F}}_{p}\right)
$$

associated with $\mathrm{m}$. (Our normalization of $\rho_{\mathfrak{m}}$ is 'geometric'.) Their proof also provides a character $\chi_{\mathfrak{m}}: \Gamma_{\mathcal{K}} \rightarrow \overline{\mathbf{F}}_{p}^{\times}$corresponding to the similitude factor; see the main text.

Assume that the signature of $G$ is $(0, n)$ except at one infinite place $\tau_{0}: F \rightarrow \mathbf{C}$. Let $\ell \neq p$ be a prime such that everything is unramified at $\ell$ and $\ell$ splits over $\mathcal{K}$. Let $v$ be a finite place of $F$ dividing $\ell \neq p$, and fix an embedding $\Gamma_{F_{v}} \rightarrow \Gamma_{F}$. In particular, the restriction of $\rho_{\mathfrak{m}}$ to $\Gamma_{F_{v}}$ is unramified. All lifts of geometric Frobenius Frob $v$ at $v$ have the same image under $\rho_{\mathfrak{m}}$, and we write $\rho_{\mathfrak{m}}\left(\right.$ Frob $\left._{v}\right)$ for the image by abuse of notation. Moreover, the conjugacy class of $\rho_{\mathfrak{m}}\left(\right.$ Frob $\left._{v}\right)$ is independent of the choice of $\Gamma_{F_{v}} \rightarrow \Gamma_{F}$. Let $\alpha_{v, 1}, \ldots, \alpha_{v, n}$ be the set of generalized eigenvalues of $\rho_{\mathfrak{m}}\left(\right.$ Frob $\left._{v}\right)$. (This means we count usual eigenvalues with multiplicities being the dimensions of the corresponding generalized eigenspaces.) We say that $\rho_{\mathfrak{m}}$ is generic at $v$ if $\alpha_{v, i} \neq q_{v} \alpha_{v, j}$ for $i \neq j$, where $q_{v}$ is the size of the residue field of $v$.

The main result of Caraiani-Scholze's work [2] in this setting is the following vanishing theorem:

(C) The Author(s), 2020. Published by Cambridge University Press. This is an Open Access article, distributed under the terms of the Creative Commons Attribution licence (http://creativecommons.org/licenses/by/4.0/), which permits unrestricted re-use, distribution, and reproduction in any medium, provided the original work is properly cited. 
Theorem $1.1([2,1.5,6.3 .3])$. If $\rho_{\mathfrak{m}}$ is generic at some $v$, then $i=\operatorname{dim} X_{K}$. Namely, $H_{\mathrm{et}}^{*}\left(X_{K, \bar{E}}, \overline{\mathbf{F}}_{p}\right)_{\mathrm{m}}$ vanishes outside the middle degree.

Remark 1.2. In [2], $\rho_{\mathrm{m}}$ is assumed to be decomposed generic at $v$ : a slightly stronger condition than being generic. But their proof can be modified easily to cover generic ones; see also [3], especially the proof of Corollary 5.1.3.

Now, assume the signature at $\tau_{0}$ is $(1, n-1)$; this is essentially the Harris-Taylor case [7]. (There are some additional technical assumptions in [7].) So, the reflex field equals $F$, and $\operatorname{dim} X_{K}=n-1$. In the Harris-Taylor case, the above vanishing theorem is also proved in [1, 4.7] by a different argument. In fact, Boyer proved the following stronger result. Note first that the Galois action on $H_{\mathrm{ett}}^{i}\left(X_{K, \bar{F}}, \overline{\mathbf{F}}_{p}\right)_{\mathfrak{m}} \otimes \chi_{\mathrm{m}}$ is unramified at $v$.

Theorem 1.3 ([1]). If $\alpha$ is an eigenvalue of Frob $_{v}$ acting on the cohomology $H_{\mathrm{et}}^{i}\left(X_{K, \bar{F}}, \overline{\mathbf{F}}_{p}\right)_{\mathfrak{m}} \otimes \chi_{\mathrm{m}}$, then the multiset

$$
\alpha, \quad q_{v} \alpha, \ldots, \quad q_{v}^{(n-1)-i} \alpha
$$

is a subset of the multiset $\left\{\alpha_{v, 1}, \ldots, \alpha_{v, n}\right\}$ of generalized eigenvalues of the action $\rho_{\mathfrak{m}}\left(\operatorname{Frob}_{v}\right)$.

Remark 1.4. There is an immediate variant using a finite extension of $F$ : let $F^{\prime}$ be a finite extension of $F$, and let $v^{\prime}$ be a finite place of $F^{\prime}$ above $v$. Then the theorem holds with Frob ${ }_{v}$ and $q_{v}$ replaced by Frob $_{v^{\prime}}$ and $q_{v^{\prime}}$. Indeed, it follows from the theorem since Frob $v_{v^{\prime}}=\operatorname{Frob}_{v}^{\left[k_{v^{\prime}}: k_{v}\right]}$ and $q_{v^{\prime}}=q_{v}^{\left[k_{v^{\prime}}: k_{v}\right]}$.

Later, the theorem or the variant above is used in the following way, combined with the Chebotarev density: let $g$ be an element of $\rho_{\mathfrak{m}}\left(\Gamma_{F^{\prime}}\right)$. Then there exist infinitely many finite places $v^{\prime}$ (respectively, $v$ ) of $F^{\prime}$ (respectively, $F$ ) to which the variant can be applied, and the conjugacy class of $g$ equals that of $\rho_{\mathfrak{m}}\left(\right.$ Frob $\left._{v^{\prime}}\right)$. Moreover, we can make $q_{v^{\prime}}=p_{v^{\prime}}=\left.v^{\prime}\right|_{\mathbf{Q}}$ as only such $v^{\prime}$ contribute to nonzero Dirichelet density (this makes $p_{v^{\prime}}=p_{v}:=\left.v\right|_{\mathbf{Q}}$ split in $\mathcal{K}$, and we can apply the results above).

Finally, note that if $F^{\prime}$ contains $F\left(\zeta_{p}\right)$, then $q_{v^{\prime}} \equiv 1 \bmod p$ for $v^{\prime}$ prime to $p$, and the statement of the variant simplifies. This simplification is very useful and will be used frequently.

Remark 1.5. Theorem 1.3 is not clearly stated in [1] but follows from an argument along the line of [1, $4.14]$ by considering the greatest integer $i^{\prime} \geq 0$ such that $H_{\mathrm{et}}^{(n-1)-i^{\prime}}\left(X_{K, \bar{F}}, \overline{\mathbf{F}}_{p}\right)_{\mathfrak{m}} \neq 0$ or (in fact, and, a posteriori) $H_{\mathrm{et}}^{(n-1)-i^{\prime}}\left(X_{K, \bar{F}}, \overline{\mathbf{F}}_{p}\right)_{\mathfrak{m}^{\vee}} \neq 0$, where $\mathrm{m}^{\vee}$ is the 'dual' of $\mathrm{m}$. It can be proved using the method of [2] as well. This will be discussed in a forthcoming article by the author.

Remark 1.6. Assume that $\ell$ splits completely in $F$. Then any eigenvalue of Frobenius at $v$ acting on $H_{\text {ét }}^{i}\left(X_{K}, \overline{\mathbf{F}}_{p}\right)_{\mathfrak{m}} \otimes \chi_{\mathfrak{m}}$ is a Frobenius eigenvalue of $\left.\rho_{\mathfrak{m}}\right|_{\Gamma_{F v}}$ by Wedhorn's congruence relation [11] and our normalization of $\rho_{\mathfrak{m}}$.

As part of a mod $p$ analog of the Arthur-Kottwitz conjecture, one would consider hypothetical Lefschetz operators $H_{\mathrm{et}}^{j}\left(X_{K, \bar{F}}, \overline{\mathbf{F}}_{p}\right)_{\mathfrak{m}} \rightarrow H_{\mathrm{et}}^{j+2}\left(X_{K, \bar{F}}, \overline{\mathbf{F}}_{p}\right)_{\mathfrak{m}}(1)$ inducing isomorphisms $H_{\mathrm{et}}^{(n-1)-i}\left(X_{K, \bar{F}}, \overline{\mathbf{F}}_{p}\right)_{\mathfrak{m}} \cong H_{\mathrm{et}}^{(n-1)+i}\left(X_{K, \bar{F}}, \overline{\mathbf{F}}_{p}\right)_{\mathfrak{m}}(i)$. This would imply that each $\alpha, \ldots, \ell^{i} \alpha$ is a Frobenius eigenvalue of $\rho_{\mathfrak{m}}$. Theorem 1.3 is stronger and gives information about multiplicities; this may also be regarded as part of the mod $p$ analog of the Arthur-Kottwitz conjecture.

The main result of this note, which is deduced from Boyer's result, is the following:

Theorem 1.7. Let $X_{K}$ be Harris-Taylor's Shimura variety of dimension $n-1$ [7]. Let $\mathrm{m}$ be a maximal ideal of the Hecke algebra contributing to the cohomology of $X_{K}$ and $\rho_{\mathfrak{m}}: \Gamma_{F} \rightarrow \mathrm{GL}_{n}\left(\overline{\mathbf{F}}_{p}\right)$ be the associated Galois representation. If $\rho_{\mathfrak{m}}$ is irreducible, then $H_{\mathrm{et}}^{j}\left(X_{K, \bar{F}}, \overline{\mathbf{F}}_{p}\right)_{\mathfrak{m}}$ vanishes for $j<n / 2$ and $j>2(n-1)-n / 2$.

In particular, the cohomology localized at $m$ vanishes outside the middle degree if $\rho_{\mathfrak{m}}$ is irreducible and $n \leq 3$. While the case $n=4$ is difficult as $n$ is no longer prime, we can push the argument further if $n=5$ : 
Theorem 1.8. Suppose $n=5$ and $\rho_{\mathfrak{m}}$ is irreducible. Then $H_{\mathrm{et}}^{*}\left(X_{K, \bar{F}}, \overline{\mathbf{F}}_{p}\right)_{\mathfrak{m}}$ vanishes outside the middle degree, except possibly when $p=5$ and every minimal noncentral normal subgroup of $\rho_{\mathfrak{m}}\left(\Gamma_{F\left(\zeta_{p}\right)}\right)$ is an elementary abelian $r$-group with $r=2$ or 3 .

Remark 1.9. For instance, the above excludes a case where $p=5$ and $\rho_{\mathfrak{m}}\left(\Gamma_{F\left(\zeta_{p}\right)}\right)$ is the semidirect product of $\{\operatorname{diag}( \pm 1, \ldots, \pm 1)\}$ (of order 32) and a permutation of order 5. (Thanks to the referee for pointing out an erroneous claim in the first version of the manuscript.)

There are previous works in this direction, including Shin [10] (see also [5]), Emerton-Gee [4], and Boyer [1]. The novelty here is that we only assume the irreducibility of $\rho_{\mathfrak{m}}$. (Let us also mention that Boyer is recently claiming a rather strong vanishing result.) For the proofs, we use Theorem 1.3 and also group-theoretic results from [6], which, in full generality, rely on the classification of finite simple groups.

It is easy to control $\rho_{\mathfrak{m}}$ with a large image. Let us record the following remark. The argument passing to $F\left(\zeta_{p}\right)$ is very important throughout this note and will also be used frequently later.

Theorem 1.10. If the image $\rho_{\mathfrak{m}}\left(\Gamma_{F\left(\zeta_{p}\right)}\right)$ of $\Gamma_{F\left(\zeta_{p}\right)}$ contains a regular semisimple element of $\mathrm{GL}_{n}\left(\overline{\mathbf{F}}_{p}\right)$, then $H_{\mathrm{et}}^{*}\left(X_{K, \bar{F}}, \overline{\mathbf{F}}_{p}\right)_{\mathfrak{m}}$ vanishes outside the middle degree.

Proof. Let $g \in \rho_{\mathfrak{m}}\left(\Gamma_{F\left(\zeta_{p}\right)}\right)$ denote a regular semisimple element of $\mathrm{GL}_{n}\left(\overline{\mathbf{F}}_{p}\right)$. We shall apply Theorem 1.3, Remark 1.4 to $g$. So, we choose a prime-to- $p$ finite place $v^{\prime}$ of $F^{\prime}=F\left(\zeta_{p}\right)$, as in Remark 1.4. In particular, $g$ belongs to the conjugacy class of $\rho_{\mathfrak{m}}\left(\right.$ Frob $\left._{v^{\prime}}\right)$. Hence, $\rho_{\mathfrak{m}}\left(\right.$ Frob $\left._{v^{\prime}}\right)$ is also regular semisimple. Observe that the multiset of eigenvalues of $\rho_{\mathfrak{m}}\left(\right.$ Frob $\left._{v^{\prime}}\right)$ does not contain a subset of the form of $\left\{\alpha, q_{v^{\prime}} \alpha\right\}$ as $q_{v^{\prime}} \equiv 1 \bmod p$. Therefore, $H_{\text {ét }}^{*}\left(X_{K, \bar{F}}, \overline{\mathbf{F}}_{p}\right)_{\mathfrak{m}}$ vanishes outside the middle degree by Remark 1.4.

Example 1.11. Suppose $n$ is an odd prime, $p>2 n-3$, and the restriction of $\rho_{\mathfrak{m}}$ to $\Gamma_{F\left(\zeta_{p}\right)}$ is irreducible: that is, $\rho_{\mathfrak{m}}$ is irreducible and not induced from a character. Then $[6,1.6]$ says that the image of $\Gamma_{F\left(\zeta_{p}\right)}$ contains a regular semisimple element. So, $H_{\mathrm{ett}}^{*}\left(X_{K, \bar{F}}, \overline{\mathbf{F}}_{p}\right)_{\mathrm{m}}$ vanishes outside the middle degree by Theorem 1.10.

Example 1.12. Another example satisfying the assumption of Theorem 1.10 is the case where the image of $\Gamma_{F}$ contains $\mathrm{SL}_{n}\left(\mathbf{F}_{p}\right)$. (Note that $\mathrm{SL}_{n}\left(\mathbf{F}_{p}\right)$ contains a regular semisimple element.) Indeed, if $(n, p) \neq(2,2),(2,3), \mathrm{SL}_{n}\left(\mathbf{F}_{p}\right)$ is perfect and contained in the image of $\Gamma_{F\left(\zeta_{p}\right)}$. If $p=2$, then $F=F\left(\zeta_{p}\right)$, and there is nothing to prove. If $p=3,\left[F\left(\zeta_{p}\right): F\right]$ divides 2 , and $\mathrm{SL}_{2}\left(\mathbf{F}_{3}\right)$ does not have a subgroup of index 2 , so it is contained in the image of $\Gamma_{F\left(\zeta_{p}\right)}$.

We also remark that Theorem 1.3 implies the following:

\section{Proposition 1.13. Suppose}

- $\rho_{\mathfrak{m}}$ is irreducible and induced from a character of $\Gamma_{E}$ for some cyclic extension $F \subset E$ of degree $n$ contained in $F\left(\zeta_{p}\right)$, and

$\circ\left[F\left(\zeta_{p}\right): F\right]>n$.

Then $H_{\mathrm{et}}^{*}\left(X_{K, \bar{F}}, \overline{\mathbf{F}}_{p}\right)_{\mathrm{m}}$ vanishes outside the middle degree.

Proof. Suppose $H_{\text {ét }}^{i}\left(X_{K, \bar{F}}, \overline{\mathbf{F}}_{p}\right)_{\mathfrak{m}}$ is nonzero for some $i<n-1$. Pick a generator $h \in \operatorname{Gal}\left(F\left(\zeta_{p}\right) / F\right)$. It maps to a generator of the quotient $\operatorname{Gal}(E / F)$ as well. We can write $h$ as the (geometric) Frobenius of some finite place $v$ of $F$ such that $q_{v}=p_{v}$. (In particular, $p_{v}$ splits in $\mathcal{K}$.) Let $\sigma \in \Gamma_{F_{v}}$ denote a lift of Frob $_{v}$; so $\sigma$ maps to $h \in \operatorname{Gal}\left(F\left(\zeta_{p}\right) / F\right)$. As $h$ maps to a generator of $\operatorname{Gal}(E / F)$, the characteristic polynomial of $\rho_{\mathfrak{m}}(\sigma)$ has the form $X^{n}-c$. Combined with Theorem 1.3, this implies that $q_{v}^{n} \equiv 1$ $\bmod p$. However, as $h$ is a generator of $\operatorname{Gal}\left(F\left(\zeta_{p}\right) / F\right) \subset(\mathbf{Z} / p \mathbf{Z})^{\times}$and $\left[F\left(\zeta_{p}\right): F\right]>n$, we have $q_{v}^{n} \equiv p_{v}^{n} \not \equiv 1 \bmod p$.

The dual argument settles the case $i>n-1$. 


\section{Preliminaries}

\subsection{Setting}

Let $F=F^{+} \mathcal{K}$ be a $\mathrm{CM}$ field with a totally real field $F^{+}$and an imaginary quadratic field $\mathcal{K}$. We fix an embedding $\mathcal{K} \hookrightarrow \mathbf{C}$. We consider a PEL datum $(B, *, V,(\cdot, \cdot))$ of type A such that

$\circ \quad B$ is a division algebra with center $F$ and $V \cong B$, and

- The associated group $G$ has signature $(1, n-1)$ at one infinite place and $(0, n)$ at the other infinite places, where $n^{2}=\operatorname{dim}_{F} B$. (The signature is calculated using the fixed embedding $\mathcal{K} \hookrightarrow \mathbf{C}$.)

Fix a sufficiently small open compact subgroup $K=\prod_{\ell} K_{\ell}$ of $G\left(\mathbb{A}_{f}\right)$. If $\ell$ splits in $\mathcal{K}$, by choosing a place $y$ of $\mathcal{K}$ over $\ell$, we have an isomorphism $G\left(\mathbf{Q}_{\ell}\right) \cong \mathbf{Q}_{\ell}^{\times} \times \prod_{x} B_{x}^{\text {op } \times}$, where $x$ runs over the places of $F$ lying over $y$.

Let $\mathrm{Spl}^{\mathrm{ur}}$ denote the set of unramified places $v$ of $F$ satisfying

$\circ v$ does not divide $p$.

$\circ p_{v}=\left.v\right|_{\mathbf{Q}}$ splits in $\mathcal{K}$.

$\circ B$ splits at all places above $p_{v}$.

$\circ K_{p_{v}}$, as a subgroup of $\mathbf{Q}_{p_{v}}^{\times} \times \prod_{x} B_{x}^{\mathrm{op} \times}$, decomposes into a product of $\mathbf{Z}_{p_{v}}^{\times}$and maximal open compact subgroups $K_{x}$ of $B_{x}^{\text {op } \times}$.

Let $\mathbb{T}$ denote the Hecke algebra

$$
\bigotimes_{p_{v} \in \mathrm{Spl}^{\mathrm{ur}} \mid \mathbf{Q}} \mathbf{Z}\left[G\left(\mathbf{Q}_{p_{v}}\right) / / K_{p_{v}}\right]
$$

If we identify $K_{v}$ with $\mathrm{GL}_{n}\left(\mathcal{O}_{F_{v}}\right)$, its factor at $v$ is generated by

$$
T_{v, j}=K_{v} \operatorname{diag}(\underbrace{p_{v}^{-1}, \ldots, p_{v}^{-1}}_{j}, \underbrace{1, \ldots, 1}_{n-j}) K_{v} .
$$

We write $c_{v}$ for the element of $\mathbb{T}$ determined by $p_{v}^{-1} \in \mathbf{Q}_{p_{v}}^{\times}$. Our choice of the Hecke operators is different from [11], [4], [1], and [2].

We denote by $X_{K}$ the canonical model of the Shimura variety attached to $(B, *, V,(\cdot, \cdot))$ of level $K$, which is a smooth projective variety over $F$. (We use the convention that [a disjoint union of copies of] the canonical model admits a usual moduli interpretation.) The mod $p$ cohomology of $X_{K, \bar{F}}$ is naturally a module of $\mathbb{T} \times G_{F}$.

Theorem 2.1 ([2, 6.3.1]). Let $\mathrm{m}$ be a maximal ideal of $\mathbb{T}$ such that, for some $i, H_{\mathrm{et}}^{i}\left(X_{K, \bar{F}}, \overline{\mathbf{F}}_{p}\right)_{\mathfrak{m}} \neq 0$. Then there is a (unique) semisimple Galois representation $\rho_{\mathfrak{m}}: \Gamma_{F} \rightarrow \operatorname{GL}_{n}\left(\overline{\mathbf{F}}_{p}\right)$ and a character $\chi_{\mathfrak{m}}: \Gamma_{\mathcal{K}} \rightarrow \overline{\mathbf{F}}_{p}^{\times}$, both unramified at $v \in \mathrm{Spl}^{\mathrm{ur}}$, such that the characteristic polynomial of $\rho_{\mathfrak{m}}\left(\right.$ Frob $\left._{v}\right)$ for $v$ is given by

$$
\sum_{j=0}^{n}(-1)^{j} q_{v}^{j(j-1) / 2} \bar{T}_{v, j} X^{n-j}
$$

and $\chi_{\mathfrak{m}}\left(\right.$ Frob $\left._{p_{v}}\right)=\bar{c}_{v}^{-1}$, where $\bar{T}_{v, j}$ and $\bar{c}_{v}$ denote the image of $T_{v, j}$ and $c_{v}$ in $\mathbb{T} / \mathfrak{m} \cong \overline{\mathbf{F}}_{p}$, respectively.

Proof. The existence of $\rho_{\mathfrak{m}}$ is proved in [2] and [1] up to normalization; our $\rho_{\mathfrak{m}}$ is a twist of the dual of the representation they constructed. The existence of $\chi_{\mathrm{m}}$ can be proved by the same method. Namely, we find a characteristic 0 lift $\Pi$ of $\mathrm{m}$ at first; $\Pi$ is a $C$-algebraic cuspidal automorphic representation of $G$, and its stable base change is a $C$-algebraic isobaric automorphic representation of $\mathcal{K}^{\times} \times \mathrm{GL}_{n}(F)$ of the form of $\psi \otimes \Pi^{1}$. The first factor $\psi$ gives rise to a character $\widetilde{\chi}_{\mathfrak{m}}^{-1}: \Gamma_{\mathcal{K}} \rightarrow \overline{\mathbf{Q}}_{p}^{\times}$via class field theory. 
(The Artin map is normalized so that uniformizers correspond to lifts of geometric Frobenius.) The reciprocal of the reduction $\bmod p$ of $\widetilde{\chi}_{\mathrm{m}}^{-1}$ is $\chi_{\mathrm{m}}$.

Throughout this note, we regard $\chi_{\mathfrak{m}}$ as a character of $\Gamma_{F}\left(\subset \Gamma_{\mathcal{K}}\right)$ as well.

\subsection{The Congruence Relation}

The congruence relation is not logically needed (in the sense that Theorem 1.3 is stronger), but we give a short explanation to clarify our notation and conventions. For every $v \in \mathrm{Spl}^{\mathrm{ur}}$, there is a canonical integral model $\mathfrak{X}_{K}$ of $X_{K}$, which is smooth and projective over $\mathcal{O}_{F_{v}}$. The action of $\mathbb{T} \times \Gamma_{F_{v}}$ passes to the mod $p$ cohomology of the special fiber $X_{K, \overline{k(v)}}$ of the canonical integral model. In particular, the Galois action on $H_{\text {ét }}^{i}\left(X_{K, \bar{F}}, \overline{\mathbf{F}}_{p}\right)$ is unramified at $v$.

Assume that $p_{v}$ splits completely in $F$. If we look at the Frobenius action on $H_{\text {ét }}^{i}\left(X_{K, \bar{F}}, \overline{\mathbf{F}}_{p}\right)_{\mathfrak{m}} \otimes \chi_{\mathfrak{m}}$, the main result of [11] implies the following relation:

$$
\sum_{j=0}^{n}(-1)^{j} q_{v}^{j(j-1) / 2} T_{v, j} \operatorname{Frob}_{v}^{n-j}=0 .
$$

The formula is stated incorrectly (or imprecisely) in [4, 3.3.1] and [1, 4.2]:

- The Hecke correspondence in [11] is a left action (as a correspondence), while the Hecke action on the Shimura variety is a right action. This is why we change the choice of the Hecke operator.

- The twist by $\chi_{\mathrm{m}}$ is needed.

\section{Proof of Theorem 1.7}

Suppose $H_{\text {et }}^{i}\left(X_{K, \bar{F}}, \overline{\mathbf{F}}_{p}\right)_{\mathfrak{m}} \neq 0$ for some $i<n / 2$, and let $\rho$ be an irreducible constituent of $H_{\text {et }}^{i}\left(X_{K, \bar{F}}, \overline{\mathbf{F}}_{p}\right)_{\mathfrak{m}} \otimes \chi_{\mathfrak{m}}$ as a representation of $\Gamma_{F}$.

Suppose that $\rho$ is a character $\chi$. Then, by Theorem 1.3 and Remark $1.4, \chi(g) \in \overline{\mathbf{F}}_{p}^{\times}$for any $g \in \Gamma_{F\left(\zeta_{p}\right)}$ appears in the set of generalized eigenvalues of $\rho_{\mathfrak{m}}(g)$ with multiplicity $\geq n-i$. Therefore, $\left(\rho_{\mathfrak{m}} \otimes \chi^{-1}\right)(g)$ has a generalized eigenvalue 1 with multiplicity $\geq n-i$. Since $n-i>n / 2$, it contradicts the following theorem. (This discussion also works for $i=n / 2$.)

Theorem 3.1 ([6, 1.5.(a)]). Let $H \subset \mathrm{GL}_{n}\left(\overline{\mathbf{F}}_{p}\right)$ be a finite group whose action on $\overline{\mathbf{F}}_{p}^{n}$ is irreducible. For any nontrivial normal subgroup $H^{\prime}$ of $H$, there exists semisimple $h \in H^{\prime}$ such that the multiplicity of 1 in the set of eigenvalues of $h$ is less than $n / 2$.

Proof. In [6], this is stated with $H^{\prime}=H$. The proof actually finds $h$ in any given minimal normal subgroup $N$ of $H$.

Suppose $\operatorname{dim} \rho \geq 2$. Then we claim that $\left.\rho\right|_{\Gamma_{F\left(\zeta_{p}\right)}}$ is not unipotent modulo scalar: namely, $\rho(h)$ is not unipotent modulo scalar for some $h \in \Gamma_{F}\left(\zeta_{p}\right)$. Indeed, assume that $\rho(h)$ is unipotent modulo scalar for every $h \in \Gamma_{F\left(\zeta_{p}\right)}$. Set $\bar{H}:=\rho\left(\Gamma_{F\left(\zeta_{p}\right)}\right) /($ scalar); this is a $p$-group because the order of any element is a power of $p$. If $\bar{H}$ is nontrivial, $\bar{H}$ has a nontrivial center $Z$. If $\widetilde{Z}$ denotes the inverse image of $Z$ in $\rho\left(\Gamma_{F\left(\zeta_{p}\right)}\right)$, then $\widetilde{Z}$ is abelian. (Consider the Jordan decomposition of elements of $\widetilde{Z}$.) Moreover, the restriction of $\rho$ to $\widetilde{Z}$ is semisimple since $\widetilde{Z}$ is normal in $\rho\left(\Gamma_{F\left(\zeta_{p}\right)}\right)$ and $\rho\left(\Gamma_{F\left(\zeta_{p}\right)}\right)$ is normal in $\rho\left(\Gamma_{F}\right)$. This is impossible as $\widetilde{Z}$ contains an element of order $p$. Thus $\bar{H}$ is trivial: that is, $\rho$ is scalar on $\Gamma_{F}\left(\zeta_{p}\right)$. Then $\rho\left(\Gamma_{F}\right)$ is abelian as $F\left(\zeta_{p}\right)$ is a cyclic extension over $F$. Contradiction.

So, there exists $h \in \Gamma_{F\left(\zeta_{p}\right)}$ such that $\rho(h)$ has at least two distinct eigenvalues, say $\alpha$, $\beta$. Each has multiplicity $\geq n-i$ in the multiset of generalized eigenvalues of $\rho_{\mathfrak{m}}(h)$. Thus, $\operatorname{dim} \rho_{\mathfrak{m}} \geq 2(n-i)>n$. Contradiction. 
If $i>2(n-1)-n / 2$, the vanishing of $H_{\text {ét }}^{i}\left(X_{K, \bar{F}}, \overline{\mathbf{F}}_{p}\right)_{\mathfrak{m}}$ follows from the vanishing of $H_{\text {ét }}^{2(n-1)-i}\left(X_{K, \bar{F}}, \overline{\mathbf{F}}_{p}\right)_{\mathfrak{m}^{\vee}}$ and the Poincaré duality because $\rho_{\mathfrak{m}^{\vee}}$ is also irreducible.

\section{Proof of Theorem 1.8}

We may only consider cohomology below the middle degree because the duality preserves the condition that $\rho_{\mathfrak{m}}$ is irreducible (and the exceptional case stated in Theorem 1.8).

Suppose that $H_{\text {ét }}^{i}\left(X_{K, \bar{F}}, \overline{\mathbf{F}}_{p}\right)_{\mathfrak{m}} \neq 0$ for some $i<4$. We will consider two cases:

(1) The restriction of $\rho_{\mathfrak{m}}$ to $\Gamma_{F\left(\zeta_{p}\right)}$ is irreducible.

(2) The restriction of $\rho_{\mathfrak{m}}$ to $\Gamma_{F\left(\zeta_{p}\right)}$ is not irreducible.

\subsection{Group-Theoretic Results}

We will use another group-theoretic result from [6]:

Theorem 4.1 ([6, 1.7]). Let $H$ be a finite non-abelian simple group and $p$ be a prime number. Assume that $(H, p) \neq\left(\mathfrak{A}_{5}, 5\right)$. Then there exist $p^{\prime}$-elements $x, y, z \in H$ with $x y z=1$ such that $H=\langle x, y\rangle$.

This will be combined with Scott's lemma:

Theorem 4.2 ([9]). Let $H$ be a finite group acting on a finite-dimensional vector space $V$ over a field $k$. Assume that $x, y, z$ generate $H$ and satisfy $x y z=1$. Then

$$
\operatorname{dim} V+\operatorname{dim} V^{H}+\operatorname{dim}\left(V^{\vee}\right)^{H} \geq \operatorname{dim} V^{x}+\operatorname{dim} V^{y}+\operatorname{dim} V^{z},
$$

where $V^{*}$ denotes the space of fixed vectors under the action of $*$.

\subsection{Preliminary Analysis}

Before dealing with case (1), let us first analyze a slightly more general situation where $n$ is a prime and $\left.\rho_{\mathfrak{m}}\right|_{\Gamma_{F\left(\zeta_{p}\right)}}$ is irreducible. The discussion below partly follows the referee's suggestion.

Let $N$ be a minimal noncentral normal subgroup of $\rho_{\mathfrak{m}}\left(\Gamma_{F\left(\zeta_{p}\right)}\right)$. We make the following hypothesis throughout Section 4.2:

\section{$N$ is not a quasi-simple group.}

We shall show that $\rho_{\mathfrak{m}}\left(\Gamma_{F\left(\zeta_{p}\right)}\right)$ contains a regular semisimple element in the following cases:

(i) The restriction of $\rho_{\mathfrak{m}}$ to $N$ is irreducible.

(ii) The restriction of $\rho_{\mathfrak{m}}$ to $N$ is not irreducible, and $n \neq p$.

(iii) The restriction of $\rho_{\mathfrak{m}}$ to $N$ is not irreducible, $n=p=5$, and $N$ is not an elementary abelian $r$-group for $r=2,3$.

Hence, Theorem 1.10 gives the vanishing outside the middle degree in these cases.

Let us first observe that the hypothesis above implies that $N$ is not perfect in all cases: if $N$ is perfect, then $N$ is non-abelian, and $\left.\rho_{\mathfrak{m}}\right|_{N}$ is a faithful irreducible representation of $N$ of prime dimension. However, this cannot happen because $N$ modulo the center $Z\left(\rho_{\mathfrak{m}}\left(\Gamma_{F\left(\zeta_{p}\right)}\right)\right)$ of $\rho_{\mathfrak{m}}\left(\Gamma_{F\left(\zeta_{p}\right)}\right)$ is the direct product of (more than one) isomorphic simple group as $N / Z\left(\rho_{\mathfrak{m}}\left(\Gamma_{F\left(\zeta_{p}\right)}\right)\right)$ is a minimal normal subgroup of $\rho_{\mathfrak{m}}\left(\Gamma_{F\left(\zeta_{p}\right)}\right) / Z\left(\rho_{\mathfrak{m}}\left(\Gamma_{F\left(\zeta_{p}\right)}\right)\right)$.

Therefore, $[N, N]$ is central in $\rho_{\mathfrak{m}}\left(\Gamma_{F\left(\zeta_{p}\right)}\right)$ by the minimality of $N$. Hence, $N$ is nilpotent. Using the minimality again, we see that $N$ is an $r$-group for some prime $r$. Let us now study each case.

(i) Assume $\left.\rho_{\mathfrak{m}}\right|_{N}$ is irreducible. In particular, $N$ is a non-abelian nilpotent $r$-group. As $\left.\rho_{\mathfrak{m}}\right|_{N}$ is irreducible and $n$ is prime, we see that $n=r$ and $p \neq n$. In fact, $\left.\rho_{\mathfrak{m}}\right|_{N}$ is induced from a character. 
So, $N$ contains a regular semisimple element by $[6,5.2]$. (Note that $n$ is assumed to be odd in the reference, but it is not used in the argument of $[6,5.2]$.)

(ii) Assume $\left.\rho_{\mathfrak{m}}\right|_{N}$ is not irreducible and $n \neq p$. Then $\left.\rho_{\mathfrak{m}}\right|_{N}$ is the direct sum of distinct characters as $N$ is a noncentral normal subgroup of $\rho_{\mathfrak{m}}\left(\Gamma_{F\left(\zeta_{p}\right)}\right)$. So, $\left.\rho_{\mathfrak{m}}\right|_{\Gamma_{F\left(\zeta_{p}\right)}}$ is induced from a character of a subgroup of $\rho_{\mathfrak{m}}\left(\Gamma_{F\left(\zeta_{p}\right)}\right)$ containing $N$, and $\rho_{\mathfrak{m}}\left(\Gamma_{F\left(\zeta_{p}\right)}\right)$ contains a regular semisimple element by $[6,5.2]$.

(iii) Assume $\left.\rho_{\mathfrak{m}}\right|_{N}$ is not irreducible, $n=p=5$, and $N$ is not an elementary abelian $r$-group for $r=2,3$. Note that $N$ is a subgroup of the diagonal $\left(\overline{\mathbf{F}}_{5}^{\times}\right)^{5}$ stable under a permutation $\tau$ of order 5, so $N$ is an elementary abelian $r$-group for the prime $r \geq 7$ by the minimality of $N$. (It suffices to observe that $N$ contains a non-scalar element of order $r$ : take a non-scalar element $x \in N$ such that $x^{r}$ is a scalar. Then $\tau(x) x^{-1}$ is a nontrivial element of order $r$, and it is not a scalar since $\tau$ has order $n$ and $n=p \neq r$.) We claim that there is a regular semisimple element inside $N$.

Let us prove the claim. From now on, we identify $N$ with a nontrivial subrepresentation of $\mathbf{Z} / 5 \mathbf{Z}$ acting on $\mathbf{F}_{r}^{5}$. If $r \not \equiv 4 \bmod 5$, then the complement of the trivial representation in $\mathbf{F}_{r}^{5}$ is either irreducible or the direct sum of four distinct characters, and it is easy to find a regular semisimple element as $r \geq 7$.

If $r \equiv 4 \bmod 5$, then the complement of the trivial representation is the direct sum of two irreducible two-dimensional subrepresentations. An element $x$ of each subrepresentation can be written as the component-wise trace of $\left(a, a \zeta_{5}, a \zeta_{5}^{2}, a \zeta_{5}^{3}, a \zeta_{5}^{4}\right)$ for $a \in \mathbf{F}_{r^{2}}$ and a choice of $\zeta_{5}$. It is easy to see that $x$ corresponds to a regular semisimple element if and only if all coordinates are distinct, if and only if $\bar{a} / a \notin\left\{1, \zeta_{5}, \zeta_{5}^{2}, \zeta_{5}^{3}, \zeta_{5}^{4}\right\}$, where $\bar{a}$ denotes the conjugate of $a$. Any norm 1 element in $\mathbf{F}_{r^{2}}$ has the form $\bar{a} / a$. Since the number of norm 1 elements in $\mathbf{F}_{r^{2}}$ is $r+1$, and $r+1>5$ by the assumption, we can find a regular semisimple $x$.

\subsection{The Case (1)}

Now $n=5$, and assume $\left.\rho_{\mathfrak{m}}\right|_{\Gamma_{F(\zeta p)}}$ is irreducible as in (1). By the discussion on (i)-(iii) above with Theorem 1.10, we only need to consider the case where some minimal noncentral normal subgroup $N$ of $\rho_{\mathfrak{m}}\left(\Gamma_{F\left(\zeta_{p}\right)}\right)$ is quasi-simple. Note that $N$ acts irreducibly on $\rho_{\mathfrak{m}}$. Let $\rho_{0}$ be an irreducible constituent of $H_{\text {ét }}^{i}\left(X_{K, \bar{F}}, \overline{\mathbf{F}}_{p}\right)_{\mathrm{m}} \otimes \chi_{\mathrm{m}}$ as a representation of $\Gamma_{F}$. We regard $\rho_{0}$ as a representation of $N$; this is possible by $[4,4.1 .3]$. (The action of $\Gamma_{F}$ on $\rho_{0}$ factors through $\rho_{\mathfrak{m}}\left(\Gamma_{F}\right)$.)

Now let $\rho$ be an irreducible constituent of $\rho_{0}$ as a representation of $N$. By Theorem 1.3 and Schur's lemma, the center $Z$ of $N$ acts on $\rho$ and $\rho_{\mathfrak{m}}$ by the same character. Therefore, $\rho_{\mathfrak{m}} \otimes \rho^{\vee}$ becomes a representation of $N / Z$, which is a simple non-abelian group.

Assume $N / Z \neq \mathfrak{A}_{5}$ or $p \neq 5$. Suppose first that $\rho$ is not isomorphic to $\left.\rho_{\mathfrak{m}}\right|_{N}$. Then we can apply Theorem 4.1 and Scott's lemma (Theorem 4.2), and there is an element $n_{0}$ of $N$ satisfying the following conditions:

$\circ n_{0}$ is a $p^{\prime}$-element. In particular, the action of $n_{0}$ on $\rho_{\mathfrak{m}} \otimes \rho^{\vee}$ is semisimple.

$\circ \operatorname{dim}\left(\rho_{\mathfrak{m}} \otimes \rho^{\vee}\right)^{n_{0}} \leq(5 \operatorname{dim} \rho) / 3$.

(Note that $Z$ is a prime-to- $p$ group since $Z \subset N \subset \rho_{\mathfrak{m}}\left(\Gamma_{F}\right)$ consists of scalars.) But this contradicts

Lemma 4.3. $\operatorname{dim}\left(\rho_{\mathfrak{m}} \otimes \rho^{\vee}\right)^{n_{0}} \geq 2 \operatorname{dim} \rho$.

Proof. Let $\lambda_{1}, \ldots, \lambda_{\operatorname{dim} \rho}$ denote the eigenvalues of $\rho\left(n_{0}\right)$, and write $v_{\lambda, i}$ for an eigenvector corresponding to $\lambda_{i}$ so that it forms a basis of $\rho$. The dual basis is denoted by $v_{\lambda, i}^{\vee}$. Theorem 1.3 and Remark 1.4 imply that, for each $i, \rho_{\mathfrak{m}}\left(n_{0}\right)$ has eigenvalue $\lambda_{i}$ with multiplicity $\geq 2$ since $n_{0} \in N \subset \rho_{\mathfrak{m}}\left(\Gamma_{F\left(\zeta_{p}\right)}\right)$. Take two linearly independent eigenvectors $w_{i, 1}, w_{i, 2}$ of $\rho_{\mathfrak{m}}\left(n_{0}\right)$ with eigenvalue $\lambda_{i}$. The following $2 \operatorname{dim} \rho$ vectors

$$
w_{1,1} \otimes v_{\lambda, 1}^{\vee}, w_{1,2} \otimes v_{\lambda, 1}^{\vee}, \ldots, w_{\operatorname{dim} \rho, 1} \otimes v_{\lambda, \operatorname{dim} \rho}^{\vee}, w_{\operatorname{dim} \rho, 2} \otimes v_{\lambda, \operatorname{dim} \rho}^{\vee}
$$

are linearly independent and fixed by $n_{0}$. 
Next, suppose $\rho$ is isomorphic to $\left.\rho_{\mathfrak{m}}\right|_{N}$. Then $\rho_{\mathfrak{m}} \otimes \rho^{\vee} \cong \operatorname{End}\left(\rho_{\mathfrak{m}}\right)$, as a representation of $N$, is selfdual and has a 1-dimensional subrepresentation and quotient representation given by the scalars and the trace map, respectively, and there is no other trivial subrepresentation or quotient representation. So, again by Theorem 4.1 and Scott's lemma (Theorem 4.2), we get an inequality $(25+2) / 3 \geq 2 \operatorname{dim} \rho=10$, which is impossible.

The only remaining case is $N / Z=\mathfrak{A}_{5}$ and $p=5$. Note that the only such quasi-simple group is $\mathfrak{A}_{5}$ itself or $\mathrm{SL}_{2}\left(\mathbf{F}_{5}\right)$, which is a double covering of $\mathfrak{A}_{5}$.

○ Suppose $N=\mathfrak{A}_{5}$. There are only three isomorphism classes of irreducible representations in characteristic 5, and one of them has dimension 5; it must be $\rho_{\mathfrak{m}}$. The other two are the trivial representation and a faithful three-dimensional representation defined over $\mathbf{F}_{5}$. Whatever $\rho$ is, any element $g$ of order 3 has an eigenvalue 1 . However, $\rho_{\mathfrak{m}}(g)$ has the eigenvalues $\left\{1, \zeta_{3}, \zeta_{3}, \zeta_{3}^{2}, \zeta_{3}^{2}\right\}$, and 1 has the multiplicity one. This contradicts Theorem 1.3 and Remark 1.4.

- Suppose $N=\mathrm{SL}_{2}\left(\mathbf{F}_{5}\right)$. Any irreducible representation in characteristic 5 is given by the symmetric power $\operatorname{Sym}^{k} \mathbf{F}_{5}^{2}$ of the standard representation of dimension 2 for an integer $k \in[0,4]$. So, $\left.\rho_{\mathfrak{m}}\right|_{N}$ must be isomorphic to $\operatorname{Sym}^{4} \mathbf{F}_{5}^{2}$. However, the central character of $\operatorname{Sym}^{4} \mathbf{F}_{5}^{2}$ is trivial; this contradicts $N \subset \rho_{\mathfrak{m}}\left(\Gamma_{F\left(\zeta_{p}\right)}\right)$.

\subsection{The Case (2)}

Again, $n=5$; and now assume that $\left.\rho_{\mathfrak{m}}\right|_{\Gamma_{F(\zeta p)}}$ is not irreducible as in (2). (In particular, 5 divides $\left[F\left(\zeta_{p}\right): F\right]$ and $p-1$.) Then $\rho_{\mathfrak{m}}$ is induced from a character, and if $\left[F\left(\zeta_{p}\right): F\right]>5$, we can apply Proposition 1.13.

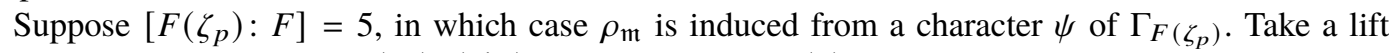
$g \in \Gamma_{F}$ of a generator of $\operatorname{Gal}\left(F\left(\zeta_{p}\right) / F\right)$; and we denote $\rho_{\mathfrak{m}}(g)$ by the same symbol, $g$. The restriction of $\rho_{\mathfrak{m}}$ to $\Gamma_{F\left(\zeta_{p}\right)}$ is the direct sum of $\psi^{g^{i}}$ for $i=0,1,2,3,4$.

Let $\rho$ be an irreducible constituent of $H_{\text {ét }}^{i}\left(X_{K, \bar{F}}, \overline{\mathbf{F}}_{p}\right)_{\mathfrak{m}} \otimes \chi_{\mathfrak{m}}$ as a representation of $\Gamma_{F}$. By [4, 4.1.3], $\rho$ may be regarded as a representation of $\rho_{\mathfrak{m}}\left(\Gamma_{F}\right)$. By Theorem 1.3, Remark 1.4, and [4, 4.1.4], the restriction of $\rho$ to $H:=\rho_{\mathfrak{m}}\left(\Gamma_{F\left(\zeta_{p}\right)}\right)$ decomposes into characters. We first show that $\rho$ itself is not a character of $\Gamma_{F}$; this implies that $\rho$ is of dimension 5 and is induced from a character of $H$.

Lemma 4.4. $\rho$ is not a character of $\Gamma_{F}$.

Proof. Suppose $\rho$ is a character $\chi$. Then $\rho_{\mathfrak{m}} \otimes \chi^{-1}$ satisfies, by Theorem 1.3 and Remark 1.4, the following condition: for any $h \in H$, the multiplicity of 1 in the multiset of eigenvalues of $\left(\rho_{\mathfrak{m}} \otimes \chi^{-1}\right)(h)$ is $\geq 2$. Up to a permutation, the multiset of eigenvalues of $\left(\rho_{\mathfrak{m}} \otimes \chi^{-1}\right)(h)$ has two possibilities:

$$
\text { (a) } 1,1, \alpha, \beta, \gamma, \quad \text { (b) } 1, \alpha, 1, \beta, \gamma, \quad \text { for some } \alpha, \beta, \gamma \neq 0 \text {. }
$$

Here, conjugation by $g$ acts by a cyclic permutation (12345).

In case (a), consideration of $\left(\rho_{\mathfrak{m}} \otimes \chi^{-1}\right)\left(h \cdot g^{2} h g^{-2}\right),\left(\rho_{\mathfrak{m}} \otimes \chi^{-1}\right)\left(h \cdot g^{3} h g^{-3}\right)$ gives $\beta=1$ or $\alpha=\gamma=1$. If $\beta=1$, consideration of $\left(\rho_{\mathfrak{m}} \otimes \chi^{-1}\right)\left(h \cdot g h g^{-1}\right),\left(\rho_{\mathfrak{m}} \otimes \chi^{-1}\right)\left(h \cdot g^{4} h g^{-4}\right)$ gives $\alpha=1$ or $\gamma=1$. So, the multiplicity of 1 is $\geq 4$ for every $h \in H$ satisfying (a) up to a permutation.

Similarly, in case (b), consideration of $\left(\rho_{\mathfrak{m}} \otimes \chi^{-1}\right)\left(h \cdot g h g^{-1}\right),\left(\rho_{\mathfrak{m}} \otimes \chi^{-1}\right)\left(h \cdot g^{4} h g^{-4}\right)$ gives $\alpha=1$ or $\beta=\gamma=1$. Since we are in case (i) as well if $\alpha=1$, we deduce that the multiplicity of 1 is $\geq 4$ for every $h \in H$. But consideration of $\left(\rho_{\mathfrak{m}} \otimes \chi^{-1}\right)\left(h \cdot g^{i} h g^{-i}\right)$ with $i \neq 0$ gives that $\left(\rho_{\mathfrak{m}} \otimes \chi^{-1}\right)(h)$ is the identity for every $h$. This is impossible.

Now we may assume that $\rho$ is induced from a character of $H$. By [4, 4.2.1] and a slight variant of [4, 4.2.4 (1)] with the same proof, we deduce that $\operatorname{det} \rho=\operatorname{det} \rho_{\mathfrak{m}}$.

Take $g^{\prime} \in H$ such that $\rho\left(g^{\prime}\right)$ is not a scalar; such an element exists because $\rho$ decomposes into distinct characters of $H$.

Lemma 4.5. Each eigenvalue of $g^{\prime}$ has multiplicity 2 or 3 in the multiset of eigenvalues of $g^{\prime}$. 
Proof. Let us first observe that $\rho(h)$ has at most two distinct eigenvalues for every $h \in H$ because if $\rho(h)$ has three distinct eigenvalues, then $h$ must have 6 eigenvalues by Theorem 1.3 and Remark 1.4.

If $\rho\left(g^{\prime}\right)$ has eigenvalues $\alpha, \alpha, \alpha, \beta, \beta$ with $\alpha \neq \beta$, then the multiplicities of $\alpha, \beta$ in the set of eigenvalues of $g^{\prime}$ are both $\geq 2$, and using the equality of determinants, we see that $\rho\left(g^{\prime}\right)$ and $g^{\prime}$ have the same characteristic polynomial. Otherwise, $\rho\left(g^{\prime}\right)$ has eigenvalues $\alpha, \alpha, \alpha, \alpha, \beta$ with $\alpha \neq \beta$. (Conjugation by $g$ acts by (12345) as before.) By applying the observation above to $\rho\left(g^{\prime 2} \cdot\left(g^{\prime}\right)^{g^{i}}\right)$ with $i \neq 0$, we see that $\alpha^{2}=\beta^{2}$ holds. Using the equality of determinants, we deduce that $g^{\prime}$ has eigenvalues $\alpha, \alpha, \beta, \beta, \beta$. This proves the lemma.

Let us complete the proof. By a permutation, we may assume that $\psi\left(g^{\prime}\right)=\psi^{g}\left(g^{\prime}\right)=\psi^{g^{2}}\left(g^{\prime}\right)$ or $\psi\left(g^{\prime}\right)=\psi^{g}\left(g^{\prime}\right)=\psi^{g^{3}}\left(g^{\prime}\right)$. In the former case, $g^{\prime} \cdot g^{2} g^{\prime} g^{-2}$ has an eigenvalue $\psi\left(g^{\prime}\right)^{2}$ with multiplicity one. In the latter case, $g^{\prime} \cdot g g^{\prime} g^{-1}$ has an eigenvalue $\psi\left(g^{\prime}\right)^{2}$ with multiplicity one. These contradict Lemma 4.5 with $g^{\prime}$ replaced by the corresponding elements as $\rho\left(g^{\prime} \cdot g^{2} g^{\prime} g^{-2}\right), \rho\left(g^{\prime} \cdot g g^{\prime} g^{-1}\right)$ are not scalars.

Acknowledgements. I thank Tetsushi Ito for helpful conversations. I am grateful to the anonymous referee for pointing out several errors and making suggestions that greatly improved the text. The main result was first presented at 'Arithmetic Geometry in Carthage' held in June 2019, where I had conversations with Ana Caraiani and Pascal Boyer on this topic. I am grateful to the organizers for providing such an opportunity. This work was supported by JSPS KAKENHI Grant Number 20 K14284.

Conflict of Interest: None.

\section{References}

[1] Pascal Boyer, 'Sur la torsion dans la cohomologie des variétés de Shimura de Kottwitz-Harris-Taylor', J. Inst. Math. Jussieu 18 (2019), no. 3, 499-517, DOI 10.1017/s1474748017000093. MR3936639.

[2] Ana Caraiani and Peter Scholze, 'On the generic part of the cohomology of compact unitary Shimura varieties', Ann. of Math. (2) 186 (2017), no. 3, 649-766, DOI 10.4007/annals.2017.186.3.1. MR3702677

[3] Ana Caraiani and Peter Scholze, 'On the generic part of the cohomology of non-compact unitary Shimura varieties', https://arxiv.org/abs/1909.01898.

[4] Matthew Emerton and Toby Gee, 'p-adic Hodge-theoretic properties of étale cohomology with mod p coeffcients, and the cohomology of Shimura varieties', Algebra Number Theory 9 (2015), no. 5, 1035-1088, DOI 10.2140/ant.2015.9.1035. MR3365999

[5] Kazuhiro Fujiwara, 'Galois deformations and arithmetic geometry of Shimura varieties', in International Congress of Mathematicians Vol. II (Eur. Math. Soc., Zürich, 2006), 347-371.

[6] Robert Guralnick and Gunter Malle, 'Characteristic polynomials and fixed spaces of semisimple elements, Recent developments in Lie algebras, groups and representation theory', in Proc. Sympos. Pure Math., vol. 86 (Amer. Math. Soc., Providence, RI, 2012), 173-186, DOI 10.1090/pspum/086/1417.

[7] Michael Harris and Richard Taylor, 'The geometry and cohomology of some simple Shimura varieties', in Annals of Mathematics Studies, vol. 151 (Princeton University Press, Princeton, NJ, 2001).

[8] Kai-Wen Lan and Junecue Suh, 'Vanishing theorems for torsion automorphic sheaves on compact PEL-type Shimura varieties', Duke Math. J. 161 (2012), no. 6, 1113-1170, DOI 10.1215/00127094-1548452.

[9] Leonard L. Scott, 'Matrices and cohomology', Ann. of Math. (2) 105 (1977), no. 3, 473-492, DOI 10.2307/1970920.

[10] Sug Woo Shin, 'Supercuspidal part of the mod 1 cohomology of GU(1; n - 1)-Shimura varieties', J. Reine Angew. Math. 705 (2015), 1-21, DOI 10.1515/crelle-2013-0057.

[11] Torsten Wedhorn, 'Congruence relations on some Shimura varieties', J. Reine Angew. Math. 524 (2000), 43-71, DOI $10.1515 /$ crl1.2000.060. 